\title{
Analysis of an Alternating Priority Queue with General Decreasing Service and with Setup Times or Server Vacations
}

\author{
Tsuyoshi KATAYAMA \\ Dept. of Electronics \& Informatics,Toyama Prefectural University \\ 5180 Kurokawa, Kosugi-machi, Imizu-gun, Toyama, Japan 939-03
}

\begin{abstract}
$\boldsymbol{A b s t r a c t ~ A n ~ a n a l y s i s ~ i s ~ p r o v i d e d ~ f o r ~ a n ~ a l t e r n a t i n g ~ p r i o r i t y ~ q u e u e ~ w i t h ~ g e n e r a l ~ d e c r e a s i n g ~ s e r v i c e ~ o f ~ a ~}$ parameter $\left(k_{1}=\infty, k_{2}=K\right)$ which operates as follows: Starting once a service of class-1 messages a single server serves all messages in queue 1 until it becomes empty. After service completion in queue 1 , the server switches over to queue 2 and continues serving messages in queue 2 until either queue 2 becomes empty, or the number of messages decreases to $K$ less than that found upon its arrival at queue 2 , whichever occurs first, where $1 \leq K \leq$ $\infty$. Two modified models with server vacations are analyzed by a uniform method. We assume that arrival streams are Poissonian, message service times for each priorty class, setup times and vacation times are generally distributed, and switch-over times are zero. This paper analyzes joint queue-length distributions and message waiting time distributions. An explicit expression is obtained for the individual mean waiting times of two-class messages. This results are applicable to the performance analysis of the register insertion ring systems in LANs.
\end{abstract}

Keywords General decreasing* service, alternating priority queue, vacation time, setup time.

\section{Introduction}

There have been some analytical studies on priority queueing systems with controllable parameters, e.g. Refs $[3,4,7]$, which are applicable to the modeling of some computer and communication systems. These priority disciplines have some advantages over the ordinary priority schedules without controllable parameters, since these enable us to control efficiently service qualities in telecommunication systems, e.g. delay time, loss probabilty etc. From such a point of view, these flexible priority disciplines are effective for the performance optimization and applicable to message processings and routing schemes in telecommunication systems. As such flexible priority disciplines, a multi-class priority disciplines with general decreasing services is proposed as follows [8]: Let there be $N$ classes of messages (or calls), where messages with a smaller class number have a nonpreemptive priority over messages with a greater class number. The service discipline is parameterized by a vector $\left(k_{1}, k_{2}, \cdots, k_{N}\right)$, where $1 \leq k_{i} \leq \infty, i=1,2, \cdots, N$. For the moment, suppose that class- $i$ messages are served, $i=1$, $2, \cdots, N$. A single server continues serving messages in queue $i$ until either queue $i$ becomes

\footnotetext{
* "decrementing" used in [8,13] is not a proper English word [M.F. Neuts 1994 (private communication)].
} 
empty, or the number of messages decrease to $k_{i}$ less than that found upon its arrival at queue $i$, whichever occurs first. We call this service discipline a general decreasing service [13] or a $k_{i}$ decreasing service. If there are messages in the system at service completion of the class- $i$ messages, messages of the highest class present in the system, say class- $j$, will be served next according to the $k_{j}$ - decreasing service. If there is no message in the system, the server waits for a new arrival. The class- $i$ service discipline with a parameter $k_{i}=1$ corresponds to the (pure) decreasing service $[9,12]$, also called the semi-exhaustive service $[2,6]$, whereas if $k_{i}=\infty$, the service discipline to the well-known exhaustive service. If $k_{i}=\infty$ for $i=1,2,3, \cdots, N$, then this priority discipline reduces to the exhaustive priority discipline treated in [13]. In the case of $N=2$ (i.e. $k_{1}=k_{2}=\infty$ ), it also reduces to the alternating priority discipline [11].

In this paper, we analyze an alternating priority queue with general decreasing service with a parameter $\left(k_{1}=\infty, k_{2}=K\right)$, where $1 \leq K<\infty$ and $K=\infty$ and with setup times or vacation times, which is a special case of the above multi-class priority queueing model and a generalized model of the alternating priority queue $[8,11]$. This results are applicable to the performance analysis of the register insertion ring systems used in local area networks (LANs) [1, 5]. In the ring system, each node has a shift register in which data received from the ring are buffered. The address portion of a received data packet (message) is read, and if the packet is addressed to the local node, the packet is removed from the ring. If the packet is addressed to another node, then it is shifted back onto the ring from the shift register. When the shift register is emptied, the local node begins to transmit its own packet from the output buffer while synchronously buffering new coming data in the shift register. When the node finishes transmitting its own packet, it then continues to shift data onto the ring from the shift register. The packets in the shift register and the output buffer are transmitted alternately.

There have been fruitful results relating to the decreasing service: Takagi [12] derived the mean message waiting time in a symmetric polling system with decreasing services of a parameter $\left(k_{1}=k_{2}=\cdots=k_{N}=1\right)$. Cohen [2] analyzed a two queue model with semi-exhaustive alternating service of a parameter $\left(k_{1}=k_{2}=1\right)$ by using the boudary value technique. Katayama [6] analyzed a single-server, two-stage tandem queue with semi-exhaustive service in the first stage and exhaustive service in the second stage, which is parameterized by $\left(k_{1}=1, k_{2}=\infty\right)$. However, there are not so many results for the general decreasing service. Takagi treated an $M / G / 1$ vacation model with general decreasing service $\left(k_{1}=K\right)$ [13], and Katayama [8] analyzed a two-class priority queue with general decreasing service of a parameter $\left(k_{1}=\infty, k_{2}=\right.$ $K<\infty)$ without setup times and vacation times.

The rest of this paper is organized as follows: Section 2 describes an alternating priority queue with general decreasing service and introduces some notations. Section 3 determines generating functions for joint queue-length distributions. Section 4 derives the Laplace-Stieltjes transforms (LSTs) of message waiting time distributions and some inequlities for the mean waiting times, and gives some numerical results. Section 5 gives concluding remarks. 


\section{Alternating Priority Queue with $K$-Decreasing Service}

This section presents a two-class priority queueing model with general decreasing service of a parameter $\left(k_{1}=\infty, k_{2}=K\right)$, where $1 \leq K \leq \infty$ and introduces some notations. The queueing system consists of two-parallel queues, $Q_{1}$ and $Q_{2}$, for messages of class- 1 and class2 , respectively. Each queue $Q_{n}$ with infinite buffer capacity has a service counter for class- $n$ messages $S_{n}, n=1,2$. Messages of class- $n$ arrive in $Q_{n}$ according to a Poisson process with rate $\lambda_{n}, n=1,2$. A service time $\tau_{n}$ for class- $n$ messages has a general distribution $H_{n}(t)$ with finite first and second moments $h_{n}$ and $h_{n}{ }^{(2)}$. The $L S T$ of $H_{n}(t)$ are denoted by $H_{n}^{*}(s), n=1,2$. Messages in $Q_{1}$ and $Q_{2}$ are served by a single server in accordance with the following priority discipline : After switching over to $S_{1}$, the server serves all messages in $Q_{1}$ until it becomes empty, i.e. the exhaustive service $\left(k_{1}=\infty\right)$. Just after service completion at $S_{1}$, the server switches over to $S_{2}$ and continues serving messages in $Q_{2}$ until either it becomes empty, or the number of messages decreases to $K$ less than that found upon its arrival at $S_{2}$, whichever occurs first, i.e. the $K$ - decreasing service $\left(k_{2}=K\right)$. Class- 1 messages are served next, if any is present. The switch-over time needed to switch service from one class to another is assumed to be zero. Messages within a class are served in each queue on the first-in-first-out (FIFO) discipline.

We will study two modified models with server vacations:

Model I : The server requires a class-dependent vacation time (setup time [7]) $\sigma_{n}$ having a general distribution $S_{n}(t)$ with finite first and second moments $s_{n}$ and $s_{n}{ }^{(2)}$, before starting a busy period with an initial message of class- $n, n=1,2$. Even if class-1 messages arrive during a setup time $\sigma_{2}$, the server starts first servicing of the class- 2 messages according to the $K$ decreasing service at $S_{2}$, as soon as its setup time terminates. The $L S T$ of $S_{n}(t), n=1,2$ is denoted by $S_{n}^{*}(s)$.

Model II : The server begins a vacation if there is no waiting message in the queueing system. The vacation may be repeated if the server finds that $Q_{1}$ and $Q_{2}$ are still empty upon his return (called a multiple vacation model [13]). If any, the server starts serving messages in $Q_{1}$ with priority over $Q_{2}$ in accordance with the decreasing service with $\left(k_{1}=\infty, k_{2}=K\right)$. A vacation time $\omega$ has a general distribution $V(t)$ with finite first and second moments $v$ and $v^{(2)}$. The $L S T$ of $V(t)$ is denoted by $V^{*}(s)$. (See subsection 4.2 and Eqs. (48.a) and (48.b)).

We introduce the following notation:

$$
\begin{array}{lll}
r_{n}:=\lambda_{n} / \lambda & n=1,2 & \lambda:=\lambda_{1}+\lambda_{2} \\
\rho_{n}:=\lambda_{n} h_{n} \quad n=1,2 & \rho:=\rho_{1}+\rho_{2} \\
h:=r_{1} h_{1}+r_{2} h_{2} \quad h^{(2)}:=r_{1} h_{1}{ }^{(2)}+r_{2} h_{2}{ }^{(2)} \\
s:=r_{1} s_{1}+r_{2} s_{2} \quad s^{(2)}:=r_{1} s_{1}{ }^{(2)}+r_{2} s_{2}{ }^{(2)} .
\end{array}
$$


For simplicity, we also define as, $Q_{3}:=Q_{1}, \lambda_{3}:=\lambda_{1}, h_{3}{ }^{(2)}:=h_{1}{ }^{(2)}, \rho_{3}:=\rho_{1}$ and so on. The total traffic intensity is assumed to be less than unity $(\rho<1)$ to ensure stability (see Remark 2.1).

We denote by $q_{n}(i)$ the probability that $i$ messages arrive at $Q_{n}$ during a service time $\tau_{n}$, $n=1,2$. We also denote by $s_{n}(i, j)$ and $v(i, j)$ the probability that $i$ messages arrive at $Q_{1}$ and $j$ messages arrive at $Q_{2}$ during a setup time $\sigma_{n}, n=1,2$ and a vacation time $\omega$, respectively. Denoting by $Q_{n}(x), S_{n}(x, y)$ and $V(x, y)$ the generating functions for $q_{n}(i), s_{n}(i, j)$ and $v(i, j)$, respectively, we have

$$
\begin{array}{ll}
Q_{n}(x)=H^{*}{ }_{n}\left\{\lambda_{n}(1-x)\right\} & n=1,2 \\
S_{n}(x, y)=S^{*}{ }_{n}\left\{\lambda_{1}(1-x)+\lambda_{2}(1-y)\right\} & n=1,2 \\
V(x, y)=V^{*}\left\{\lambda_{1}(1-x)+\lambda_{2}(1-y)\right\} . &
\end{array}
$$

We denote by $B_{n}(t)$ and $B_{n}^{*}(s)$ a busy period distribution at $S_{n}$ and the $L S T$ of $B_{n}(t), n=1,2$, respectively. We also denote by $g_{n}(i ; m)$ the probability that $i$ messages arrive at $Q_{n+1}$ during an $m$-busy period at $S_{n}$, which corresponds to a busy period started with $m$ messages at $S_{n}$, and by $G_{n}(x ; m)\left(:=\sum_{i=0}^{\infty} g_{n}(i ; m) x^{i}\right)$ the generating function for $g_{n}(i ; m)$. From the busy-period analysis in the standard $M / G / 1$ queue, we then have

$$
\begin{aligned}
& B_{n}^{*}(s)=H_{n}^{*}\left\{s+\lambda_{n}\left(1-B_{n}^{*}(s)\right)\right\} \\
& G_{n}(x):=G_{n}(x ; 1)=B_{n}^{*}\left\{\lambda_{n+1}(1-x)\right\} \quad n=1,2
\end{aligned}
$$

and

$$
G_{n}(x ; m)=\left\{G_{n}(x)\right\}^{m} \quad m=1,2,3, \cdots<\infty \quad n=1,2 .
$$

The following abbreviations are used for a differentiable generating function $G_{n}(x, y)$,

$$
G_{n x}^{\prime}(a, b):=\left[\frac{\partial}{\partial x} G_{n}(x, y)\right]_{x=a, y=b} \quad G_{n x y}^{\prime \prime}(a, b):=\left[\frac{\partial^{2}}{\partial x \partial y} G_{n}(x, y)\right]_{x=a, y=b .}
$$

Remark 2.1 The present model I (II) and the ordinary $M / G / 1$ queue with arrival rate $\lambda$, service time distribution $r_{1} H_{1}(t)+r_{2} H_{2}(t)$ and setup time distribution $r_{1} S_{1}(t)+r_{2} S_{2}(t)$ ( vacation time distribution $V(t)$ ), have the same distribution of the number of messages served in a busy period and the same non-busy time distribution.

\section{Queueing Analysis}

This section considers two kinds of joint queue-length distributions in $Q_{1}$ and $Q_{2}$ for the alternating priority queue with $K$ - decreasing service.

\subsection{Queue-Length Generating Function at Service-Completion Points}

First, let us assume that $K$ is finite, i.e. $1 \leq K<\infty$, through this subsection. We need to determine a set of generating function for joint queue-length distribution at service-completion points at $S_{1}$ and $S_{2}$. We introduce: 
$\phi_{n}(i, j)$ : the steady-state probability that $i$ messages are waiting in $Q_{1}$ and $j$ messages are waiting in $Q_{2}$ just after service-completion points at $S_{n}, n=1,2, i, j=0,1,2, \cdots$, that is, when the server has completed the exhaustive service at $S_{1}$ or the $K$-decreasing service at $S_{2}$

and

$$
\Phi_{n}(x, y):=\sum_{i=0}^{\infty} \sum_{j=0}^{\infty} \phi_{n}(i, j) x^{i} y^{j} \quad|x|,|y| \leq 1 \quad n=1,2 .
$$

Then, considering the events that occur during two successive service-completion points at the service counters $S_{1}$ and $S_{2}$, we then get the following functional relationship for Model (I) with setup times:

where

$$
\begin{aligned}
\Phi_{1}(0, y)= & \phi_{0} r_{1} x S_{1}\left(G_{1}(y), y\right) \frac{G_{1}(y)}{x}+\Phi_{2}\left\{G_{1}(y), y\right\}-\Phi_{2}(0, y) \\
\Phi_{2}(x, y)= & \phi_{0} r_{2} y \widetilde{S}_{K}\left(x, G_{2}(x)\right) \frac{G_{2}(x)}{y}+\phi_{0} r_{2} y\left\{S_{2}(x, y)-\widetilde{S}_{K}(x, y)\right\} \frac{G_{2}(x)^{K}}{y^{K}} \\
& +\sum_{j=1}^{K-1} \phi_{j} G_{2}(x)^{j}+\left\{\Phi(y)-\sum_{j=0}^{K-1} \phi_{j} y^{j}\right\} \frac{G_{2}(x)^{K}}{y^{K}}
\end{aligned}
$$

$$
\begin{aligned}
& \widetilde{S}_{K}(x, y):=\sum_{i=0}^{\infty} \sum_{j=0}^{K-1} s_{2}(i, j) x^{i} y^{j} \\
& \phi_{j}:=\phi_{1}(0, j)+\phi_{2}(0, j) \quad j=0,1,2, \cdots \\
& \Phi(y):=\Phi_{1}(0, y)+\Phi_{2}(0, y) .
\end{aligned}
$$

Similarly, for Model (II) with vacation times, we get

$$
\begin{aligned}
\Phi_{1}(0, y)= & \phi_{0} \sum_{m=1}^{\infty} v(0,0)^{m-1}\left\{V\left(G_{1}(y), y\right)-V(0, y)\right\}+\Phi_{2}\left\{G_{1}(y), y\right\}-\Phi_{2}(0, y) \\
\Phi_{2}(x, y)= & \phi_{0} \sum_{m=1}^{\infty} v(0,0)^{m-1}\left[\widetilde{V}_{K}\left(0, G_{2}(x)\right)-V(0,0)+\left\{V(0, y)-\widetilde{V}_{K}(0, y)\right\} \frac{G_{2}(x)^{K}}{y^{K}}\right] \\
& +\sum_{j=1}^{K-1} \phi_{j} G_{2}(x)^{j}+\left\{\Phi(y)-\sum_{j=0}^{K-1} \phi_{j} y^{j}\right\} \frac{G_{2}(x)^{K}}{y^{K}}
\end{aligned}
$$

where

$$
\widetilde{V}_{K}(0, y):=\sum_{j=0}^{K-1} v(0, j) y^{j} .
$$

As a general expression, we thus get the following functional equations:

$$
\begin{aligned}
& \Phi_{1}(0, y)=\phi_{0} a(y)+\Phi_{2}\left\{G_{1}(y), y\right\}-\Phi_{2}(0, y) \\
& \Phi_{2}(x, y)=\phi_{0} b_{K}(x, y)+\Phi(y) \frac{G_{2}(x)^{K}}{y^{K}}+\sum_{j=1}^{K-1} \phi_{j}\left\{G_{2}(x)^{j}-y^{j-K} G_{2}(x)^{K}\right\}
\end{aligned}
$$


where, for Model (I) with setup times,

$$
\begin{aligned}
& a(y):=r_{1} G_{1}(y) S_{1}\left(G_{1}(y), y\right) \\
& b_{K}(x, y):=r_{2} G_{2}(x) \widetilde{S}_{K}\left(x, G_{2}(x)\right)-\frac{G_{2}(x)^{K}}{y^{K}}+r_{2} y\left\{S_{2}(x, y)-\widetilde{S}_{K}(x, y)\right\} \frac{G_{2}(x)^{K}}{y^{K}}
\end{aligned}
$$

and, for Model (II) with vacation times,

$$
\begin{aligned}
& a(y):=\frac{1}{1-v(0,0)}\left\{V\left(G_{1}(y), y\right)-V(0, y)\right\} \\
& b_{K}(x, y):=\frac{1}{1-v(0,0)}\left[\widetilde{V}_{K}\left(0, G_{2}(x)\right)-v(0,0)+\left\{V(0, y)-\widetilde{V}_{K}(0, y)-1+v(0,0)\right\} \frac{G_{2}(x)^{K}}{y^{K}}\right] .
\end{aligned}
$$

It is necessary to determine the unknown functions $G_{n}(y), n=1,2, \Phi(y)$ and the unknown probabilities $\phi_{j}, j=0,1, \cdots, K-1$ on the right-hand sides of (8.a) and (8.b).

(1) Determination of $G_{n}(x), n=1,2$

Under the condition $\rho_{n} \leq 1, n=1,2$, from (3.a) and Takacs' lemma [10], an explicit expression for $G_{n}(x)$ is given by

$$
G_{n}(x)=\sum_{j=1}^{\infty} \int_{0}^{\infty} \frac{\left(\lambda_{n} t\right)^{j-1}}{j !} e^{-\left(\lambda_{n}+\lambda_{n+1}(1-x)\right) t} d H_{n}^{(j)}(t) \quad n=1,2
$$

where $H_{n}^{(j)}(t)$ denotes the $j$-fold convolution of $H_{n}(t)$ with itself, $n=1,2$.

(2) Determination of $\phi_{j}, j=1, \cdots, K-1$

Eliminating $\Phi_{2}\left\{G_{1}(y), y\right\}$ from (8.a) and (8.b) after setting $x=G_{1}(y)$ in (8.b), we get

$$
\begin{aligned}
\Phi(y)=\frac{y^{K}}{y^{K}-G_{2}\left\{G_{1}(y)\right\}^{K}} & {\left[\phi_{0}\left\{a(y)+b_{K}\left(G_{1}(y), y\right)\right\}\right.} \\
+ & \left.\sum_{j=1}^{K-1} \phi_{j}\left\{G_{2}\left\{G_{1}(y)\right\}^{j}-y{ }^{j-K} G_{2}\left\{G_{1}(y)\right\}^{K}\right\}\right] .
\end{aligned}
$$

It is shown by Takacs' lemma that the denominator on the right-hand side of (12), $y^{K}-G_{2}\left\{G_{1}(y)\right\}^{K}$ has exactly $(K-1)$ zeros $y=\omega_{r}, r=1,2, \cdots, K-1$, in the unit circle $|y|<1$ under the condition $\rho \leq 1$ (see Appendix). By the regularity of $\Phi(y)$, the numerator on the righthand side of (12) should be equal to zero for $y=\omega_{r}, r=1,2, \cdots, K-1$. We thus get the following simultaneous linear equations for $\phi_{j}, j=1,2, \cdots, K-1$ :

$$
\sum_{j=1}^{K-1} \alpha_{j}\left(\omega_{r}\right) \phi_{j}=\alpha_{0}\left(\omega_{r}\right) \phi_{0} \quad r=1,2, \cdots, K-1
$$

where 


$$
\begin{aligned}
& \alpha_{0}\left(\omega_{r}\right):=a\left(\omega_{r}\right)+b_{K}\left(G_{1}\left(\omega_{r}\right), \omega_{r}\right) \\
& \alpha_{j}\left(\omega_{r}\right):=\omega_{r}{ }^{j}-G_{2}\left\{G_{1}\left(\omega_{r}\right)\right\}^{j} \quad j=1,2, \cdots, K-1 .
\end{aligned}
$$

Using Cramer's formula, $\phi_{j}, j=1,2, \cdots, K-1$ can be given by an expression with unknown probability $\phi_{0}$ as follows:

$$
\phi_{j}=\phi_{0} \Delta_{j} \quad \Delta_{j}:=\frac{\left|D_{j}\right|}{|D|} \quad j=1,2, \cdots, K-1
$$

where $\left|D_{j}\right|$ and $|D|$ are the determinants formed by coeffcients of the simultaneous equations (13.a), and $\Delta_{0}:=1$. The unknown probability $\phi_{0}$ in (13.a) shall be determined in (3).

\section{(3) Determination of $\Phi(y)$ and $\phi_{0}$}

Substituting $\phi_{j}=\phi_{0} \Delta_{j}, j=1,2, \cdots, K$-1 for $\phi_{j}$ on the right-hand side of (12), $\Phi(y)$ can be expressed by a form with unknown probability $\phi_{0}$. As the result, $\Phi_{1}(0, y)$ and $\Phi_{2}(x, y)$ given by (8.a) and (8.b) can also be expressed by a form with only unknown probability $\phi_{0}$. The probability $\phi_{0}$ should be determined by the normalization condition, $\Phi_{1}(0,1)+\Phi_{2}(1,1)=1$.

In this way, the generating functions $\Phi_{n}(x, y), n=1,2$ have been completely determined. We thus obtain the following results.

Theorem 1. In the case $1 \leq K<\infty$, the generating functions $\Phi_{n}(x, y), n=1,2$ for the joint queue-length distribution $\left\{\phi_{n}(i, j)\right\}$ are given by :

$$
\begin{aligned}
& \Phi_{1}(0, y)=\phi_{0}\left[a(y)+\Phi\left\{G_{1}(y), y\right\}-\Phi(0, y)\right] \\
& \Phi_{2}(x, y)=\phi_{0} \Phi(x, y)
\end{aligned}
$$

where

$$
\begin{aligned}
& \Phi(x, y):=b_{K}(x, y)+\frac{G_{2}(x)^{K}}{y^{K}-G_{2}\left\{G_{1}(y)\right\}^{K}} \sum_{j=1}^{K-1} \Delta_{j}\left[G_{2}\left(G_{1}(y)\right)^{j}-y^{j-K} G_{2}\left(G_{1}(y)\right)^{K}\right] \\
& \quad+\frac{G_{2}(x)^{K}}{y^{K}-G_{2}\left\{G_{1}(y)\right\}^{K}}\left\{a(y)+b_{K}\left(G_{1}(y), y\right)\right\}+\sum_{j=1}^{K-1} \Delta_{j}\left\{G_{2}(x)^{j}-y^{j-K} G_{2}(x)^{K}\right\} \\
& \phi_{0}=[a(1)+2 \Phi(1,1)-\Phi(0,1)]^{-1}
\end{aligned}
$$

$G_{n}(x), n=1,2$ and $\Delta_{j}, j=1,2, \cdots, K-1$ are given by (11) and (14), respectively.

\subsection{Queue-Length Generating Function with $K=\infty$}

Through this subsection, it is assumed that $K$ is infinite, (i.e. the exhaustive service in the service counter $S_{2}$ ). Then, we get the following functional equations corresponding to Eqs. (8.a) and (8.b) for the case $K<\infty$. 


$$
\begin{aligned}
& \Phi_{1}(0, y)=\phi_{0} a(y)+\Phi_{2}\left\{G_{1}(y), 0\right\}-\Phi_{2}(0,0) \\
& \Phi_{2}(x, 0)=\phi_{0} b(x)+\Phi\left(G_{2}(x)\right)
\end{aligned}
$$

where, for Model (I) with setup times,

$$
\begin{aligned}
& a(y):=r_{1} G_{1}(y) S_{1}\left(G_{1}(y), y\right) \\
& b(x):=r_{2} G_{2}(x) S_{2}\left(x, G_{2}(x)\right)-1
\end{aligned}
$$

and, for Model (II) with vacation times,

$$
\begin{aligned}
& a(y):=\frac{1}{1-v(0,0)}\left\{V\left(G_{1}(y), y\right)-V(0, y)\right\} \\
& b(x):=\frac{1}{1-v(0,0)}\left\{V\left(0, G_{2}(x)\right)-v(0,0)\right\}-1 .
\end{aligned}
$$

Note that $\Phi_{2}(0, y)=\Phi_{2}(0,0)$, i.e. $\Phi(y)=\Phi_{1}(0, y)+\Phi_{2}(0,0)$. Here, eliminating $\Phi_{2}\left\{G_{1}(y), 0\right\}$ from (17.a) and (17.b) after setting $x=G_{1}(y)$ in (17.b), we obtain a non-homogeneous linear functional equation for $\Phi(y)$,

$$
\Phi(y)-\Phi[f(y)]=\phi_{0} g(y)
$$

where

$$
\begin{aligned}
& f(y):=G_{2}\left(G_{1}(y)\right) \\
& g(y):=a(y)+b\left(G_{1}(y)\right) .
\end{aligned}
$$

Using an iterative scheme, e.g. [7, 11], $\Phi(y)$ can be determined as follows :

First, let us introduce a sequence of $\left\{y_{i}\right\}$ and a function defined by

and

$$
\begin{aligned}
& y_{0}=y \quad 0 \leq y \leq 1 \\
& y_{i}=f\left(y_{i-1}\right) \quad i=1,2,3, \cdots
\end{aligned}
$$

$$
g\left\{y_{i} \mid y_{0}=y\right\}:=g\left(y_{i}\right) \quad i=0,1,2, \cdots .
$$

Then, it follows from (19.a) that

$$
\Phi\left(y_{i}\right)-\Phi\left(y_{i+1}\right)=\phi_{0} g\left(y_{i}\right) \quad i=0,1,2, \cdots .
$$

Using this relationship repeatedly, we have

$$
\Phi(y)=\eta+\phi_{0} \sum_{i=0}^{\infty} g\left\{y_{i} \mid y_{0}=y\right\}
$$

where $\eta$ is a constant which is independent of the sequence of $\left\{y_{i}\right\}$. The convergence of the infinite sum in (22.a) is assured under the condition $\rho<1$ as also shown in Appendix in [7]. By a boundary condition, $\Phi(0)=\phi_{0}$, the constant $\eta$ can be determined as

$$
\eta=\phi_{0}\left[1-\sum_{i=0}^{\infty} g\left\{y_{i} \mid y_{0}=0\right\}\right]
$$


Thus, we get

$$
\Phi(y)=\phi_{0}[1-G(0)+G(y)]
$$

where

$$
G(y):=\sum_{i=0}^{\infty} g\left\{y_{i} \mid y_{0}=y\right\} .
$$

It remains to determine the probability $\phi_{0}$, which should be determined by the normalization condition, $\Phi_{1}(0,1)+\Phi_{2}(1,0)=1 . \Phi_{1}(0, y)$ and $\Phi_{2}(x, 0)$ given by (17.a) and (17.b) can be expressed by a form with only unknown probability $\phi_{0}$. We thus obtain the following result.

Theorem 2. In the case of $K=\infty$, the generating functions $\Phi_{n}(x, y), n=1,2$ for the joint queue-length distribution $\left\{\phi_{n}(i, j)\right\}$ are given by :

$$
\begin{aligned}
& \Phi_{1}(0, y)=\phi_{0}\left[a(y)+\Phi\left\{G_{1}(y), 0\right\}-\Phi(0,0)\right] \\
& \Phi_{2}(x, 0)=\phi_{0} \Phi(x, 0)
\end{aligned}
$$

where

$$
\begin{aligned}
& \Phi(x, 0):=1+b(x)-G(0)+G\left(G_{2}(x)\right) \\
& \phi_{0}=\left[1+a(1)+2 b(1)+2 G(1)-b(0)-G(0)-G\left(G_{2}(0)\right)\right]^{-1}
\end{aligned}
$$

$G_{n}(x), n=1,2$ and $G(y)$ are given by (11) and (23.b), respectively.

\subsection{Queue-Length Generating Function at Message Departure Points}

In this subsection, we analyze the queue-length distribution at departure points of messages from each service counter. Let

$\pi_{n}(i)$ : the steady-state probability that $i$ messages are waiting in $Q_{n}$ just after a class- $n$ message has completed service at the service counter $S_{n}, n=1,2, i=0,1,2, \cdots$

and

$$
\Pi_{n}(x):=\sum_{i=0}^{\infty} \pi_{n}(i) x^{i} \quad|x| \leq 1 \quad n=1,2 .
$$

We first define a conditional probability and its generating function in order to get $\Pi_{n}(x)$, $n=1,2$ :

$q_{n}(i ; m):$ the probability that $i$ messages are waiting in $Q_{n}$ just after a class- $n$ message has completed service during an $m$-busy period at $S_{n}, n=1,2, i=0,1,2, \cdots$;

and

$$
m=1,2,3, \cdots<\infty
$$

$$
Q_{n}(x ; m):=\sum_{i=0}^{\infty} q_{n}(i ; m) x^{i} \quad|x| \leq 1 \quad n=1,2 .
$$


Then, a balance equation for $\left\{q_{n}(i ; m)\right\}$ is derived as

$$
q_{n}(i ; m)=q_{n}(i-m+1)+\sum_{j=1}^{i+1} q_{n}(j ; m) q_{n}(i-j+1) \quad m=1,2,3, \cdots<\infty
$$

which leads to the generating function [8]:

$$
Q_{n}(x ; m)=\frac{Q_{n}(x)}{x-Q_{n}(x)}\left(x^{m}-1\right) \quad m=1,2,3, \cdots<\infty \quad n=1,2 .
$$

(Note that $\sum_{m=1}^{\infty} Q_{n}(1 ; m) p_{m}=1$, denoting by $p_{m}$ the probability that a busy period starts with $m$ messages.)

(1) Model (I) with Setup Times

For the case $1 \leq K<\infty$, we get

$$
\begin{aligned}
& \Pi_{1}(x)=C_{1 K} {\left[\phi_{0} r_{1} \sum_{i=0}^{\infty} \sum_{j=0}^{\infty} s_{1}(i, j) Q_{1}(x ; i+1)+\sum_{i=1}^{\infty} \sum_{j=0}^{\infty} \phi_{2}(i, j) Q_{1}(x ; i)\right] } \\
& \Pi_{2}(x)=C_{2 K}\left[\phi_{0} r_{2} \sum_{i=0}^{\infty} \sum_{j=0}^{K-1} s_{2}(i, j) Q_{2}(x ; j+1)+\phi_{0} r_{2} \sum_{i=0}^{\infty} \sum_{j=K}^{\infty} s_{2}(i, j) x^{j+1-K} Q_{2}(x ; K)\right. \\
&\left.+\sum_{j=1}^{K-1} \phi_{j} Q_{2}(x ; j)+\sum_{j=K}^{\infty} \phi_{j} x^{j-K} Q_{2}(x ; K)\right]
\end{aligned}
$$

and, for the case $K=\infty$,

$$
\begin{aligned}
& \Pi_{1}(x)=C_{1 \infty}\left[\phi_{0} r_{1} \sum_{i=0}^{\infty} \sum_{j=0}^{\infty} s_{1}(i, j) Q_{1}(x ; i+1)+\sum_{i=1}^{\infty} \phi_{2}(i, 0) Q_{1}(x ; i)\right] \\
& \Pi_{2}(x)=\text { the symmetrical form of } \Pi_{1}(x)
\end{aligned}
$$

where coefficients $C_{n K}$ and $C_{n \infty}, n=1,2$ are normalizing constants. The normalization conditions, $\Pi_{n}(1)=1, n=1,2$ lead to the constants:

$$
\begin{aligned}
& C_{1 K}^{-1}=\frac{\phi_{0}}{1-\rho_{1}}\left[r_{1}\left(1+\lambda_{1} s_{1}\right)+\Phi_{x}^{\prime}(1,1)\right] \\
& C_{2 K}^{-1}=\frac{\phi_{0}}{1-\rho_{2}}\left[r_{2}\left\{\widetilde{S}_{K}(1,1)+K\left(1-\widetilde{S}_{K}(1,1)\right)+\widetilde{S}_{K y}{ }^{\prime}(1,1)\right\}\right. \\
&\left.-K\left\{1-r_{1}-\Phi(1,1)\right\}-\sum_{j=1}^{K-1}(K-j) \Delta_{j}\right]
\end{aligned}
$$




$$
C_{n \infty}^{-1}=\frac{r_{n} \phi_{0}}{1-\rho}(1+\lambda s) \quad n=1,2 .
$$

Thus, we get the following results.

Theorem 3. The generating functions $\Pi_{n}(x), n=1,2$ for the queue-length distribution $\left\{\pi_{n}(i)\right\}$ in Model (I) with setup times are given by : For the case $1 \leq K<\infty$,

$$
\begin{aligned}
& \Pi_{1}(x)=\frac{\phi_{0} C_{1 K} Q_{1}(x)}{x-Q_{1}(x)}\left[r_{1}\left\{x S_{1}(x, 1)-1\right\}+\Phi(x, 1)-\Phi(1,1)\right] \\
& \Pi_{2}(x)=\frac{\phi_{0} C_{2 K} Q_{2}(x)}{x-Q_{2}(x)}\left[r_{2}\left\{x \widetilde{S}_{K}(1, x)-\widetilde{S}_{K}(1,1)+x\left(1-x^{-K}\right)\left(S_{2}(1, x)-\widetilde{S}_{K}(1, x)\right)\right\}\right. \\
& \left.\quad-\sum_{j=1}^{K-1}\left\{1-x^{j-K}\right\} \Delta_{j}+\frac{1-x^{K}}{x^{K}}\left\{1-r_{1} G_{1}(x) S_{1}\left(G_{1}(x), x\right)-\Phi\left(G_{1}(x), x\right)\right\}\right]
\end{aligned}
$$

where $\Phi(x, y), \Delta_{j}, j=1,2, \cdots, K-1$ and $\phi_{0} C_{n K}, n=1,2$ are given by (16.a) in Theorem 1, (14) and (31.a), respectively.

For the case $K=\infty$,

$$
\begin{aligned}
& \Pi_{1}(x)=\frac{\phi_{0} C_{1 \infty} Q_{1}(x)}{x-Q_{1}(x)}\left[r_{1}\left\{x S_{1}(x, 1)-1\right\}+\Phi(x, 0)-\Phi(1,0)\right] \\
& \Pi_{2}(x)=\frac{\phi_{0} C_{2 \infty} Q_{2}(x)}{x-Q_{2}(x)}\left[r_{2}\left\{x S_{2}(1, x)-1\right\}+a(x)+\Phi\left(G_{1}(x), 0\right)-a(1)-\Phi(1,0)\right]
\end{aligned}
$$

where $\Phi(x, 0)$ and $\phi_{0} C_{n \infty}, n=1,2$ are given by (25.c) in Theorem 2 and (31.b), respectively.

(2) Model (II) with Vacation Times

For the case $1 \leq K<\infty$, we get

$$
\begin{aligned}
& \Pi_{1}(x)=C_{1 K} {\left[\phi_{0} \sum_{m=1}^{\infty} v(0,0)^{m-1} \sum_{i=1}^{\infty} \sum_{j=0}^{\infty} v(i, j) Q_{1}(x ; i)+\sum_{i=1}^{\infty} \sum_{j=0}^{\infty} \phi_{2}(i, j) Q_{1}(x ; i)\right] } \\
& \Pi_{2}(x)=C_{2 K}\left[\phi_{0} \sum_{m=1}^{\infty} v(0,0)^{m-1}\left\{\sum_{j=1}^{K-1} v(0, j) Q_{2}(x ; j)+\sum_{j=K}^{\infty} v(0, j) x^{j-K} Q_{2}(x ; K)\right\}\right. \\
&\left.+\sum_{j=1}^{K-1} \phi_{j} Q_{2}(x ; j)+\sum_{j=K}^{\infty} \phi_{j} x^{j-K} Q_{2}(x ; K)\right]
\end{aligned}
$$

and, for the case $K=\infty$, 


$$
\begin{aligned}
& \Pi_{1}(x)=C_{1 \infty}\left[\phi_{0} \sum_{m=1}^{\infty} v(0,0)^{m-1} \sum_{i=1}^{\infty} \sum_{j=0}^{\infty} v(i, j) Q_{1}(x ; i)+\sum_{i=1}^{\infty} \phi_{2}(i, 0) Q_{1}(x ; i)\right] \\
& \Pi_{2}(x)=C_{2 \infty}\left[\phi_{0} \sum_{m=1}^{\infty} v(0,0)^{m-1} \sum_{j=1}^{\infty} v(0, j) Q_{2}(x ; j)+\sum_{j=1}^{\infty} \phi_{j} Q_{2}(x ; j)\right]
\end{aligned}
$$

where coefficients $C_{n K}$ and $C_{n \infty}, n=1,2$ are normalizing constants. The normalization conditions, $\Pi_{n}(1)=1, n=1,2$ lead to the constants:

$$
\begin{aligned}
& C_{1 K}{ }^{-1}=\phi_{0}\left[\frac{K \lambda_{1} h_{2}}{\left(1-\rho_{1}\right)\left(1-\rho_{2}\right)}+\frac{\lambda_{1} v}{\left(1-V^{*}(\lambda)\right)(1-\rho)}\right] \\
& C_{2 K}{ }^{-1}=\frac{\phi_{0}}{1-\rho_{2}}\left[\frac{1}{1-V^{*}(\lambda)}\left\{\widetilde{V}_{K y}{ }^{\prime}(0,1)-K \widetilde{V}_{K^{\prime}}(0,1)+K V(0,1)-V_{y}{ }^{\prime}(0,1)+\lambda_{1} v G_{1}{ }^{\prime}(1)+\lambda_{2} v\right\}\right. \\
& C_{n \infty}{ }^{-1}=\frac{\phi_{0}}{(1-\rho)} \frac{\lambda_{n} v}{1-V^{*}(\lambda)} \quad n=1,2 .
\end{aligned}
$$

Thus, we get the following results.

Theorem 4. The generating functions $\Pi_{n}(x), n=1,2$ for the queue-length distribution $\left\{\pi_{n}(i)\right\}$ in Model (II) with vacation times are given by: For the case $1 \leq K<\infty$,

$$
\begin{gathered}
\Pi_{1}(x)=\frac{\phi_{0} C_{1 K} Q_{1}(x)}{x-Q_{1}(x)}\left[\frac{1}{1-V^{*}(\lambda)}\{V(x, 1)-1\}+\Phi(x, 1)-\Phi(1,1)\right] \\
\Pi_{2}(x)=\frac{\phi_{0} C_{2 K} Q_{2}(x)}{x-Q_{2}(x)}\left[\frac{1}{1-V^{*}(\lambda)}\left\{x^{-K} \widetilde{V}_{K}(0, x)-x^{-K} V(0, x)-\widetilde{V}_{K}(0,1)+V\left(G_{1}(x), x\right)\right\}\right. \\
\left.\quad-\left(1-x^{-K}\right)+\Phi\left(G_{1}(x), x\right)-\sum_{j=1}^{K-1}\left(1-x^{j-K}\right) \Delta_{j}\right]
\end{gathered}
$$

where $\Phi(x, y), \Delta_{j}, j=1,2, \cdots, K-1$ and $\phi_{0} C_{n K}, n=1,2$ are given by (16.a) in Theorem 1, (14) and (36.a), respectively.

For the case $K=\infty$,

$$
\begin{aligned}
& \Pi_{1}(x)=\frac{\phi_{0} C_{1 \infty} Q_{1}(x)}{x-Q_{1}(x)}\left[\frac{1}{1-V^{*}(\lambda)}\{V(x, 1)-1\}+\Phi(x, 0)-\Phi(1,0)\right] \\
& \Pi_{2}(x)=\frac{\phi_{0} C_{2 \infty} Q_{2}(x)}{x-Q_{2}(x)}\left[\frac{1}{1-V^{*}(\lambda)}\{V(0, x)-V(0,1)\}+a(x)\right.
\end{aligned}
$$




$$
\left.+\Phi\left(G_{1}(x), 0\right)-a(1)-\Phi(1,0)\right]
$$

where $\Phi(x, 0)$ and $\phi_{0} C_{n \infty}, n=1,2$ are given by (25.c) in Theorem 2 and (36.b), respectively.

\section{Waiting Time Analysis}

This section derives closed-form expressions for waiting time distributions (LSTs) and the mean waiting times of class- $n$ messages, $n=1,2$.

\subsection{Waiting Time Distributions}

We denote by $W_{n}{ }^{*}(s)$ the $L S T$ of distribution function for the waiting time of class- $n$ messages $W_{n}(t), n=1,2$. By the usual argument that the number of class- $n$ messages at the departure time in $S_{n}$ is equal to the number of class- $n$ messages that arrive at $Q_{n}$ during its sojourn time because of the FIFO service discipline, we have

$$
W_{n}^{*}\left\{\lambda_{n}(1-x)\right\} H_{n}^{*}\left\{\lambda_{n}(1-x)\right\}=\Pi_{n}(x) \quad n=1,2 .
$$

Thus, we obtain the following result.

Theorem 5. The LSTs of waiting time distributions for class- $n$ messages, $n=1,2$ are given by

$$
W_{n}^{*}(s)=\Pi_{n}\left\{1-s / \lambda_{n}\right\} / H_{n}^{*}(s) \quad n=1,2
$$

where $\Pi_{n}(x), n=1,2$ are given by Theorem 3 (Model (I) with setup times) and Theorem 4, (Model (II) with vacation times). The $m$ th moment of the waiting time for class- $n$ messages, $n=1,2$ is calculated by

$$
E\left(W_{n}^{m}\right)=(-1)^{m} W_{n}^{*(m)}(0) \quad n=1,2 \quad m=1,2,3, \cdots .
$$

\subsection{Mean Waiting Times}

From Theorems 3, 4 and 5, we have

$$
W_{n}^{*}(s)=\frac{-\lambda_{n} \phi_{0} C_{n K}}{s-\lambda_{n}\left\{1-H_{n}^{*}(s)\right\}} F_{n}\left(1-s / \lambda_{n}\right) \quad 1 \leq K \leq \infty \quad n=1,2
$$

where $F_{n}\left(1-s / \lambda_{n}\right)$ is obtained by setting $x=1-s / \lambda_{n}$ in the numerator of $\Pi_{n}(x), n=1,2$ given by Theorem $3\left(\right.$ Model (I)) and Theorem 4 (Model (II)), respectively. Denoting by $E\left(W_{n}\right)_{K}, n=1$, 2 the mean waiting time for class- $n$ messages in the case of $K$-decreasing service, we then get

$$
E\left(W_{n}\right)_{K}=\frac{\lambda_{n} h_{n}{ }^{(2)}}{2\left(1-\rho_{n}\right)}+\frac{1}{\lambda_{n}} \frac{F_{n}{ }^{\prime \prime}(1)}{2 F_{n}^{\prime}(1)} \quad 1 \leq K \leq \infty \quad n=1,2
$$


where we have used a relationship $W_{n}^{*}(0)=1$ and applied L'Hospital's rule to (41) because of $F_{n}(1)=0$.

For special cases of $K=1$ (the pure decreasing service) and $K=\infty$ (the exhaustive service), $E\left(W_{n}\right)_{K}, n=1,2$ are expressed as follows:

Corollary 1. For Model (I) with Setup Times:

$$
\begin{aligned}
E\left(W_{1}\right)_{K=1} & =\frac{\lambda_{1} h_{1}{ }^{(2)}}{2\left(1-\rho_{1}\right)}+\frac{\lambda_{2} h_{2}^{(2)}}{2\left(1-\rho_{1}\right)\left(1-\rho_{2}\right)^{2}}+\frac{\pi_{0}}{1-\rho_{1}}\left[s_{1}+\frac{\rho_{2}}{1-\rho_{2}} s_{2}\right]+\frac{\pi_{0}}{2\left(1-\rho_{1}\right)} \lambda s^{(2)} \\
E\left(W_{2}\right)_{K=1} & =\frac{\lambda_{1} h_{1}^{(2)}}{2\left(1-\rho_{1}\right)(1-\rho)}+\frac{\lambda_{2} h_{2}{ }^{(2)}}{2\left(1-\rho_{2}\right)}\left[1+\frac{\rho_{1}{ }^{2}}{\left(1-\rho_{1}\right)\left(1-\rho_{2}\right)(1-\rho)}\right] \\
& +\frac{\pi_{0}}{\left(1-\rho_{1}\right)(1-\rho)}\left[\rho_{1} s_{1}+\frac{\rho_{1} \rho_{2}+\left(1-\rho_{1}\right)(1-\rho)}{1-\rho_{2}} s_{2}\right]+\frac{\pi_{0}}{2\left(1-\rho_{1}\right)(1-\rho)} \lambda s^{(2)}
\end{aligned}
$$

and

$$
\begin{aligned}
E\left(W_{1}\right)_{K=\infty} & =\frac{\lambda_{1} h_{1}{ }^{(2)}}{2\left(1-\rho_{1}\right)}+\frac{\rho_{2}{ }^{2} \lambda_{1} h_{1}{ }^{(2)}+\left(1-\rho_{1}\right)^{2} \lambda_{2} h_{2}{ }^{(2)}}{2\left(1-\rho_{1}\right)(1-\rho)\left(1-\rho+2 \rho_{1} \rho_{2}\right)}+\frac{\pi_{0}}{1-\rho_{1}}\left[s_{1}+\frac{\rho_{1} \rho_{2}{ }^{2} s_{1}+\left(1-\rho_{1}\right)^{2} \rho_{2} s_{2}}{(1-\rho)\left(1-\rho+2 \rho_{1} \rho_{2}\right)}\right] \\
& +\frac{\pi_{0}}{2\left(1-\rho_{1}\right)} \lambda_{1} s_{1}{ }^{(2)}+\frac{\pi_{0}}{2\left(1-\rho_{1}\right)(1-\rho)\left(1-\rho+2 \rho_{1} \rho_{2}\right)}\left\{\rho_{2}{ }^{2} \lambda_{1} s_{1}{ }^{(2)}+\left(1-\rho_{1}\right)^{2} \lambda_{2} s_{2}{ }^{(2)}\right\} \\
E\left(W_{2}\right)_{K=\infty} & =\frac{\lambda_{2} h_{2}{ }^{(2)}}{2\left(1-\rho_{2}\right)}+\frac{\left(1-\rho_{2}\right)^{2} \lambda_{1} h_{1}{ }^{(2)}+\rho_{1}{ }^{2} \lambda_{2} h_{2}{ }^{(2)}}{2\left(1-\rho_{2}\right)(1-\rho)\left(1-\rho+2 \rho_{1} \rho_{2}\right)}+\frac{\pi_{0}}{1-\rho_{2}}\left[s_{2}+\frac{\rho_{1}\left(1-\rho_{2}\right)^{2} s_{1}+\rho_{1}{ }^{2} \rho_{2} s_{2}}{(1-\rho)\left(1-\rho+2 \rho_{1} \rho_{2}\right)}\right] \\
& +\frac{\pi_{0}}{2\left(1-\rho_{2}\right)} \lambda_{2} s_{2}{ }^{(2)}+\frac{\pi_{0}}{2\left(1-\rho_{2}\right)(1-\rho)\left(1-\rho+2 \rho_{1} \rho_{2}\right)}\left\{\left(1-\rho_{2}\right)^{2} \lambda_{1} s_{1}{ }^{(2)}+\rho_{1}{ }^{2} \lambda_{2} s_{2}{ }^{(2)}\right\}
\end{aligned}
$$

where

$$
\pi_{0}:=\frac{1-\rho}{1+\lambda s} .
$$

Corollary 2. For Model (II) with Vacation Times:

$$
\begin{aligned}
& E\left(W_{1}\right)_{K=1}=\frac{\lambda_{1} h_{1}{ }^{(2)}}{2\left(1-\rho_{1}\right)}+\frac{\lambda_{2} h_{2}^{(2)}}{2\left(1-\rho_{1}\right)\left(1-\rho_{2}\right)^{2}}+\frac{1-\rho}{1-\rho_{1}} \frac{v^{(2)}}{2 v} \\
& E\left(W_{2}\right)_{K=1}=\frac{\lambda_{1} h_{1}{ }^{(2)}}{2\left(1-\rho_{1}\right)(1-\rho)}+\frac{\lambda_{2} h_{2}{ }^{(2)}}{2\left(1-\rho_{2}\right)}\left[1+\frac{\rho_{1}{ }^{2}}{\left(1-\rho_{1}\right)\left(1-\rho_{2}\right)(1-\rho)}\right]+\frac{1}{1-\rho_{1}} \frac{v^{(2)}}{2 v}
\end{aligned}
$$


and

$$
\begin{aligned}
& E\left(W_{1}\right)_{K=\infty}=\frac{\lambda_{1} h_{1}{ }^{(2)}}{2\left(1-\rho_{1}\right)}+\frac{\rho_{2}{ }^{2} \lambda_{1} h_{1}{ }^{(2)}+\left(1-\rho_{1}\right)^{2} \lambda_{2} h_{2}{ }^{(2)}}{2\left(1-\rho_{1}\right)(1-\rho)\left(1-\rho+2 \rho_{1} \rho_{2}\right)}+\frac{\rho_{2} \rho+\left(1-\rho_{2}\right)(1-\rho)}{1-\rho+2 \rho_{1} \rho_{2}} \frac{v^{(2)}}{2 v} \\
& E\left(W_{2}\right)_{K=\infty}=\frac{\lambda_{2} h_{2}{ }^{(2)}}{2\left(1-\rho_{2}\right)}+\frac{\left(1-\rho_{2}\right)^{2} \lambda_{1} h_{1}{ }^{(2)}+\rho_{1}{ }^{2} \lambda_{2} h_{2}{ }^{(2)}}{2\left(1-\rho_{2}\right)(1-\rho)\left(1-\rho+2 \rho_{1} \rho_{2}\right)}+\frac{1-\rho_{2}}{1-\rho+2 \rho_{1} \rho_{2}} \frac{v^{(2)}}{2 v} .
\end{aligned}
$$

Corollaries 1 and 2 satisfy the following pseudo-conservation laws, respectively [13],

$$
\sum_{n=1}^{2} \rho_{n} E\left(W_{n}\right)_{K} / \rho=\frac{\lambda h^{(2)}}{2(1-\rho)}+\frac{\pi_{0}}{2 \rho(1-\rho)}\left[2 \sum_{n=1}^{2} \rho_{n} s_{n}+\rho \lambda s^{(2)}\right]
$$

and

$$
\sum_{n=1}^{2} \rho_{n} E\left(W_{n}\right)_{K} / \rho=\frac{\lambda h^{(2)}}{2(1-\rho)}+\frac{v^{(2)}}{2 v} .
$$

In the case of setup times independent of the class of the first message, i.e., $s=s_{1}=s_{2}$ and $s^{(2)}=s_{1}{ }^{(2)}=s_{2}{ }^{(2)}$, there is the following relationship between the moments of setup time distribution and that of vacation time distribution as

$$
\frac{v^{(2)}}{2 v}=\frac{2 s+\lambda s^{(2)}}{2(1+\lambda s)}
$$

which is well known as the conversion equation of setup time to vacation time [13]. It should be noticed that we have

$$
E_{S}\left(W_{n}\right)_{K} \neq E_{V}\left(W_{n}\right)_{K} \quad \text { for all } K(=1,2,3, \cdots \leq \infty) \quad n=1,2
$$

even under the condition of (48.a), where we denote by $E_{S}\left(W_{n}\right)_{K}$ and $E_{V}\left(W_{n}\right)_{K}$ the mean waiting time of class- $n$ messages in Model (I) with setup times and that in Model (II) with vacation times, respectively. This is due to a slight difference of the service discipline for messages of class 1 which arrive during a setup time $\sigma_{2}$ in Model (I) and during a vacation time $\omega$ in Model (II) as described in Section 2. However, it follows from (47.a), (47.b) and (48.a) that

$$
\sum_{n=1}^{2} \rho_{n} E_{S}\left(W_{n}\right)_{K}=\sum_{n=1}^{2} \rho_{n} E_{V}\left(W_{n}\right)_{K} \quad \text { for all } K(=1,2,3, \cdots \leq \infty) .
$$

(This is also confirmed for $K=1$ and $K=\infty$ in Corollaries 1 and 2.)

\section{Remark 4.1}

(1) Setting $s_{n}=s_{n}{ }^{(2)}=0$ and $v^{(2)} / 2 v=0$ in Corollaries 1 and 2, it is confirmed that $E\left(W_{n}\right)_{K=1}$, $n=1,2$ agree with the previous results (Eqs. (21.a) and (21.b) in [8] ). Eqs. (44.a) and (44.b) are mutually symmetric functions, which have been also derived in $[7,13]$ as the special cases of the other queueing model. 
(2) It is obvious from Theorems 3,4 , and 5 that $E\left(W_{n}\right)_{K}, n=1,2$ for $2 \leq K<\infty$ are expressed by the form with $\Delta_{j}, j=1,2, \cdots, K-1$ defined by (14). Hence, the coefficients of $v^{(2)} / 2 v$ in the expression for the mean waiting times $E_{V}\left(W_{n}\right)_{K}, n=1,2$ are given by functions with the parameter $K$ as implicitly shown in Corollary 2 .

We next proceed to the study on upper and lower bounds for mean waiting times $E\left(W_{n}\right)_{K}$, $n=1$, 2. Firstly, we recall the conservation law, Eqs. (47.a) and (47.b). Comparing two service disciplines at the service counter $S_{2}$, i.e. the $K$ - decreasing service and the $(K+1)$ decreasing service, we have

$$
E\left(W_{2}\right)_{K}>E\left(W_{2}\right)_{K+1}
$$

This relationship is derived from the fact that the chance of services for class-2 messages should be monotonously increasing as the parameter $K$ increases. The inequality (49) and the relationships (47.a) and (47.b) yield the following bounds for the mean waiting times of class- $n$ messages, $n=1,2$.

Corollary 3. $E\left(W_{n}\right)_{K}, n=1,2$ for $2 \leq K<\infty$ have the following upper and lower bounds :

$$
\begin{aligned}
& E\left(W_{1}\right)_{K=1}<E\left(W_{1}\right)_{K}<E\left(W_{1}\right)_{K=\infty} \\
& E\left(W_{2}\right)_{K=1}>E\left(W_{2}\right)_{K}>E\left(W_{2}\right)_{K=\infty} .
\end{aligned}
$$

It is noticed in Model (I) with setup times that the inequality $E\left(W_{1}\right)_{K=1}<E\left(W_{2}\right)_{K=1}$ does not always hold for system parameters $\left\{\lambda_{n}, h_{n}, s_{n}, h_{n}{ }^{(2)}, s_{n}{ }^{(2)}, n=1,2\right\}$ such that $\rho<1$. From Corollary 1 for Model (I) with setup times, the necessary and sufficient conditions are derived for the inequalities $E\left(W_{1}\right)_{K=1}<E\left(W_{2}\right)_{K=1}$ and $E\left(W_{1}\right)_{K=\infty}<E\left(W_{2}\right)_{K=\infty}$, respectively, as follows:

$$
\begin{aligned}
& 2 \pi_{0}\left(1-\rho_{2}\right)\left(1-\rho_{1}-\rho\right) s_{1}+\rho\left[1-\rho_{1}-\frac{\rho_{1}}{1-\rho_{2}}\right] \lambda_{2} h_{2}{ }^{(2)} \\
< & 2 \pi_{0}\left\{\rho_{1} \rho_{2}+(1-\rho)^{2}\right\} s_{2}+\pi_{0} \rho\left(1-\rho_{2}\right)\left\{\lambda_{1} s_{1}{ }^{(2)}+\lambda_{2} s_{2}{ }^{(2)}\right\}+\rho\left(1-\rho_{2}\right) \lambda_{1} h_{1}{ }^{(2)}
\end{aligned}
$$

and

$$
\begin{aligned}
& \left(1-\rho_{1}\right)\left\{\rho_{1}\left(\rho_{2}-2 \rho_{1}\right)+(1-\rho)\left(\rho-2 \rho_{1} \rho_{2}\right)\right\} \lambda_{2} h_{2}{ }^{(2)}+2 \pi_{0}\left(1-\rho_{2}\right)\left\{(1-\rho)\left(1-\rho_{1}-\rho\right)\right. \\
& \left.+\rho_{1} \rho_{2}\left(2-3 \rho_{1}-\rho_{2}\right)\right\} s_{1}+2 \pi_{0}\left(1-\rho_{2}\right)\left\{\rho_{2}{ }^{2}-\left(1-\rho_{1}\right)\left(1-\rho_{2}\right)\right\} \lambda_{1} s_{1}{ }^{(2)} \\
< & \left(1-\rho_{2}\right)\left\{\rho_{2}\left(\rho_{1}-2 \rho_{2}\right)+(1-\rho)\left(\rho-2 \rho_{1} \rho_{2}\right)\right\} \lambda_{1} h_{1}{ }^{(2)}+2 \pi_{0}\left(1-\rho_{1}\right)\left\{(1-\rho)\left(1-\rho_{2}-\rho\right)\right. \\
& \left.+\rho_{1} \rho_{2}\left(2-\rho_{1}-3 \rho_{2}\right)\right\} s_{2}+2 \pi_{0}\left(1-\rho_{1}\right)\left\{\rho_{1}{ }^{2}-\left(1-\rho_{1}\right)\left(1-\rho_{2}\right)\right\} \lambda_{2} s_{2}{ }^{(2)}
\end{aligned}
$$

For Model (II) with vacation times, it follows similarly from Corollary 2 that

and

$$
\left[1-\rho_{1}-\frac{\rho_{1}}{1-\rho_{2}}\right] \lambda_{2} h_{2}{ }^{(2)}<\left(1-\rho_{2}\right)\left[\lambda_{1} h_{1}{ }^{(2)}+2(1-\rho) \frac{v^{(2)}}{2 v}\right]
$$




$$
\begin{aligned}
& \left(1-\rho_{1}\right)\left\{\rho_{1}\left(\rho_{2}-2 \rho_{1}\right)+(1-\rho)\left(\rho-2 \rho_{1} \rho_{2}\right)\right\} \lambda_{2} h_{2}{ }^{(2)} \\
+ & 2\left(1-\rho_{2}\right)(1-\rho)\left\{\rho_{2}{ }^{2}-\rho_{1} \rho_{2}-(1-\rho)\left(\rho-2 \rho_{1} \rho_{2}\right)\right\} \frac{v^{(2)}}{2 v} \\
< & \left(1-\rho_{2}\right)\left\{\rho_{2}\left(\rho_{1}-2 \rho_{2}\right)+(1-\rho)\left(\rho-2 \rho_{1} \rho_{2}\right)\right\} \lambda_{1} h_{1}{ }^{(2)} .
\end{aligned}
$$

From (49) and (51.a) to (52.b), we thus get the following results.

\section{Corollary 4.}

Denoting by $A$ and $B$ a set of system parameters for Model (I) with setup times $\left\{\lambda_{n}, h_{n}, s_{n}\right.$, $\left.h_{n}{ }^{(2)}, s_{n}{ }^{(2)}, n=1,2\right\}$ satisfying (51.a) and (51.b), respectively, the following relationships hold:

(1) $B \subset A$.

(2) For any element of a set $B, E\left(W_{1}\right)_{K}<E\left(W_{2}\right)_{K}$ for all $K(=1,2,3, \cdots)$.

(3) For any element of a set $(A \cap \bar{B})$, there exists a parameter $k=K, 1 \leq K<\infty$ such that $E\left(W_{1}\right)_{K}<E\left(W_{2}\right)_{K}$

(4) For any element of a set $\bar{A}$, there exists no parameter $k=K, 1 \leq K<\infty$ such that

$$
E\left(W_{1}\right)_{K}<E\left(W_{2}\right)_{K}
$$

The above relationships (1) to (4) also hold for Model (II) with vacation times, where we need to denote by $A$ and $B$ a set of system parameters $\left\{v, v^{(2)}, \lambda_{n}, h_{n}, h_{n}^{(2)}, n=1,2\right\}$ satisfying (52.a) and (52.b), respectively.

\section{Remark 4.2}

The property of (4) in Corollary 4 can be explained as follows: Messages arriving at a heavytraffic queue (queue 2 ) have a more chance that queue 2 is currently being served than those arriving at a light-traffic queue (queue 1), even in the case of $K=1$. The similar property has been also pointed out for the polling system with exhaustive service.

\subsection{Comparison of Priority Disciplines}

It is useful to clear the difference between the mean waiting time of messages of class $n$, $E\left(W_{n}\right), n=1,2$ in Corollaries 1 and 2 and the mean waiting time, $E\left(W_{n}\right)_{N P}$, for the standard nonpreemptive (head-of-the-line) priority queueing system ( $M / G / 1$-type queue) with setup times and multiple server vacations given by:

For Model (I) with setup times [7],

$$
\begin{aligned}
& E\left(W_{1}\right)_{N P}=\frac{1}{2\left(1-\rho_{1}\right)}\left\{\lambda_{1} h_{1}{ }^{(2)}+\lambda_{2} h_{2}{ }^{(2)}\right\}+\frac{\pi_{0}}{2\left(1-\rho_{1}\right)}\left[2\left(s_{1}+\rho_{2} s_{2}\right)+\lambda_{1} s_{1}{ }^{(2)}+\lambda_{2} s_{2}{ }^{(2)}\right] \\
& E\left(W_{2}\right)_{N P}=\frac{\lambda_{1} h_{1}{ }^{(2)}+\lambda_{2} h_{2}{ }^{(2)}}{2\left(1-\rho_{1}\right)(1-\rho)}+\frac{\pi_{0}\left[2\left(\rho_{1} s_{1}+\rho_{2} s_{2}\right)+2\left(1-\rho_{1}\right)(1-\rho) s_{2}+\lambda_{1} s_{1}{ }^{(2)}+\lambda_{2} s_{2}{ }^{(2)}\right]}{2\left(1-\rho_{1}\right)(1-\rho)}
\end{aligned}
$$


and, for Model (II) with multiple server vacations [13],

$$
\begin{aligned}
& E\left(W_{1}\right)_{N P}=\frac{1}{2\left(1-\rho_{1}\right)}\left\{\lambda_{1} h_{1}{ }^{(2)}+\lambda_{2} h_{2}{ }^{(2)}\right\}+\frac{1-\rho}{1-\rho_{1}} \frac{v^{(2)}}{2 v} \\
& E\left(W_{2}\right)_{N P}=\frac{\lambda_{1} h_{1}{ }^{(2)}+\lambda_{2} h_{2}{ }^{(2)}}{2\left(1-\rho_{1}\right)(1-\rho)}+\frac{1}{1-\rho_{1}} \frac{v^{(2)}}{2 v} .
\end{aligned}
$$

Table 1 shows for Model (I) with setup times that for $\rho=0.2$ to $0.9, E\left(W_{1}\right)_{N P}<E\left(W_{1}\right)_{K=1}$ $<E\left(W_{1}\right)_{K=\infty}$ and $E\left(W_{2}\right)_{N P}>E\left(W_{2}\right)_{K=1}>E\left(W_{2}\right)_{K=\infty}$, where constant setup times $s_{1}=s_{2}=1$, constant service times $h_{1}=h_{2}=1$ and $\lambda_{1} / \lambda_{2}=20$. The same characteristics of the mean waiting times in Model (II) with multiple server vacations is shown in Table 2, where constant vacation time $v=1$, constant service times $h_{1}=h_{2}=1$ and $\lambda_{1} / \lambda_{2}=20$. It is also seen from Tables 1 and 2 that $E\left(W_{1}\right)_{N P}$ in Model I (Table 1) $>E\left(W_{1}\right)_{N P}$ in Model II (Table 2) and $E\left(W_{2}\right)_{N P}$ in Model I (Table 1) $>E\left(W_{2}\right)_{N P}$ in Model II (Table 2) for the same server utiization, and that the same relationships hold for $E\left(W_{n}\right)_{K=1}$ and $E\left(W_{n}\right)_{K=\infty}, n=1,2$.

\section{Table 1}

Mean Waiting Time $E\left(W_{n}\right), n=1,2$ for Model (I ) as a function of server utilization $\rho$

\begin{tabular}{ccccccc}
\hline$\rho$ & $E\left(W_{1}\right)_{N P}$ & $E\left(W_{1}\right)_{K=1}$ & $E\left(W_{1}\right)_{K=\infty}$ & $E\left(W_{2}\right)_{N P}$ & $E\left(W_{2}\right)_{K=1}$ & $E\left(W_{2}\right)_{K-\infty}$ \\
\hline \hline 0.2 & 1.0373 & 1.0374 & 1.0377 & 1.1299 & 1.1261 & 1.1221 \\
0.4 & 1.1670 & 1.1679 & 1.1691 & 1.6593 & 1.6422 & 1.6178 \\
0.6 & 1.4750 & 1.4775 & 1.4841 & 3.3125 & 3.2629 & 3.1309 \\
0.8 & 2.3511 & 2.3583 & 2.4216 & 11.311 & 11.168 & 9.9014 \\
0.9 & 3.7000 & 3.7144 & 4.0985 & 36.526 & 36.238 & 28.557 \\
\hline
\end{tabular}

\section{Table 2}

Mean Waiting Time $E\left(W_{n}\right), n=1,2$ for Model (II) as a function of server utilization $\rho$

\begin{tabular}{ccccccc}
\hline$\rho$ & $E\left(W_{1}\right)_{N P}$ & $E\left(W_{1}\right)_{K=1}$ & $E\left(W_{1}\right)_{K=\infty}$ & $E\left(W_{2}\right)_{N P}$ & $E\left(W_{2}\right)_{K=1}$ & $E\left(W_{2}\right)_{K=\infty}$ \\
\hline \hline 0.2 & 0.6176 & 0.6178 & 0.6178 & 0.7721 & 0.7698 & 0.7681 \\
0.4 & 0.8077 & 0.8083 & 0.8091 & 1.3462 & 1.3341 & 1.3185 \\
0.6 & 1.1667 & 1.1687 & 1.1741 & 2.9167 & 2.8769 & 2.7689 \\
0.8 & 2.1000 & 2.1065 & 2.1647 & 10.500 & 10.371 & 9.2065 \\
0.9 & 3.5000 & 3.5137 & 3.8811 & 35.000 & 34.725 & 27.377 \\
\hline
\end{tabular}




\section{Concluding Remarks}

For an alternating priority queue with $K$-decreasing service and with setup times or vacation times, we have derived LSTS for waiting time distributions and explicit expressions for mean waiting times. It is further study to apply the delay cycle analysis in order to derive LSTs for waiting time distributions, directly. Further research will be also extended to the analysis of the influence of the switch-over time (overhead time) on some performance measures and to the optimization of switching rules $[3,4]$ and decreasing parameters. For example, using the results obtained here, it may be possible to determine an optimal parameter $k=k_{\text {opt }}$ so as to minimize a cost function defined by a linear combination of individual mean waiting times by help of computer programs.

\section{References}

[1] D. Bertsekas and R.G. Gallager: "Data Networks," Prentice-Hall (1987).

[2] J.W. Cohen: "A two-queue model with semi-exhaustive alternating service," Performance '87, Edited by P.-J. Courtois and G. Latouche, pp. 19-37, North-Holland (1988).

[3] S.S. Fratini: "Analysis of dynamic priority queue," Commun. Statist.-Stochastic Models, 6, 3, pp. 415-444 (1990).

[4] M. Hofri and K.W. Ross: "On the optimal control of two queues with server set-up times and its analysis," SIAM J. Comput., 16, 2, pp. 399-420 (1987).

[5] D.E. Huber, W. Steinlin and P.J. Wild: "SILK: An implementation of a buffer insertion ring," IEEE J. on Selected Areas in Communications, SAC-1, 5, pp. 766-774 (1983).

[6] T. Katayama: "A cyclic-service tandem queueing model with semi-exhaustive service," Performance of Distributed and Parallel Systems, Eds. T. Hasegawa, H. Takagi and Y. Takahashi, pp. 173-183, North-Holland (1989).

[7] T. Katayama and Y. Takahashi: "Analysis of a two-class priority queue with Bernoulli schedules," J. of the Operations Research Society of Japan, 35, 3, pp. 236-249 (1992).

[8] T. Katayama: " Performance analysis for a two-class priority queueing model with general decrementing service," IEICE Trans. Commun., E75-B, 12, pp. 1301-1307 (1992).

[9] B.G. Pittel': "Optimal control in a mass service system with several flows of demands," Engineering Cybernetics, 11, 6, pp. 1029-1044 (1972).

[10] L. Takacs: "Introduction to the Theory of Queues," Oxford University Press, New York (1962).

[11] _ : "Two queues attended by a single server," Oper. Res., 16, 3, pp. 639-650 (1968).

[12] H. Takagi: "Mean message waiting time in a symmetric polling system," Performance'84, Models of Computer System Performance, Edited by Gelenbe, pp. 293-302, North-Holland (1985). 
[13] _ : "Queueing Analysis: A Foundation of Performance Evaluation Vol. I, Vacation and Priority Systems, Part 1," Elsevier Science Publisher B. V., North-Holland (1991).

Appendix. Zeros of the denominator of Equation (12)

From (12), we have

$$
y^{K}-G_{2}\left\{G_{1}(y)\right\}^{K}=y^{K}-B^{*}\left[\lambda_{1}\left\{1-B^{*}{ }_{1}\left(\lambda_{2}(1-y)\right)\right\}\right]^{K} \text {. }
$$

Let us consider a transcendental equation for $y$,

$$
y^{K}-H^{*}\left(\lambda_{2}(1-y)\right)=0
$$

where

$$
H^{*}(s):=B_{2}^{*}\left[\lambda_{1}\left\{1-B_{1}^{*}(s)\right\}\right]^{K} .
$$

Applying Takacs' lemma [10] to (A.2), we obtain the following results :

If $\rho \leq 1$, then Eq.(A.2) has exactly $K$ roots $\omega_{r}, r=1,2, \cdots, K-1$ in the unit circle $|y|<1$, while $\omega_{K}=1$, and $\omega_{r}, r=1,2, \cdots, K-1$ are explicitly expressed by

$$
\omega_{r}:=\sum_{j=1}^{\infty} \frac{\left(-\lambda_{2}\right)^{j-1} \theta_{r}^{j}}{j !} \frac{d^{j-1}}{d \lambda_{2}^{j-1}}\left\{H^{*}\left(\lambda_{2}\right)\right\}^{j / K}
$$

where

$$
\theta_{r}:=\exp \left(\frac{2 \pi r i}{K}\right), \quad i=\sqrt{-1}, \quad r=1,2, \cdots, K-1 .
$$

